\title{
Agile in the management of scientific research collaborations
}

\author{
Kristian P Thaller \\ University of Southampton, Southampton, United Kingdom
}

Correspondence should be addressed to: Kristian P Thaller ; K.P.Thaller@soton.ac.uk

Received date: 22 April 2014; Accepted date: 20 December 2014; Published date: 7 October 2015

Academic Editor: Valentina Ndou

Copyright (C) 2015. Kristian P Thaller . Distributed under Creative Commons CC-BY 4.0

\begin{abstract}
Multidisciplinary scientific research collaborations in academic institutions remain at the center of the global innovation system. This article highlights the distinct challenges to developing an effective management strategy that such collaborations pose. These include the fundamental challenge of having the generation of new explicit knowledge as an objective and the integration of established research teams including their respective bodies of tacit knowledge. The parallels between established Agile strategies and a proposed management strategy that addresses these challenges are discussed. By considering the contextual framework for the proposed management strategy and identifying the traditional and Agile components, this article aims to provide a generalized approach that a project management professional can use to develop an effective integrated management strategy for project, program and knowledge management in scientific research collaborations that is based on recognized techniques.
\end{abstract}

Keywords: Agile; Scientific Research; Research Program; Collaboration.

\section{Introduction}

The origins and impact of collaborative research in science were first studied in detail by Beaver and Rosen (1978, 1979). In their analysis Beaver and Rosen identified a long standing trend towards increased collaboration. By the 1990s it had been recognized that this trend was continuing towards heterogeneity in the innovation sector, the increased role of non-academic institutions such as corporate research laboratories in the collaborations (e.g. Gibbons et al., 1994). The continued importance of new scientific knowledge, i.e. the high risk academic contribution, to genuinely disruptive innovation remains however clear. Stimulated by a growing governmental emphasis on delivering the economic impact of knowledge and the imperative of sourcing supplementary revenue streams, academic institutions continue to be at the center of the innovation system (e.g. Etzkowitz\& Leydesdorff,1996 and Godin \&Gingras, 2000).One effect of the evolving context for innovative research funding has been the accelerated growth of large-scale multidisciplinary collaborative research in academic institutions.Such research programs present a unique set of challenges for which there does not exist an accepted best practice management strategy. This article identifies the key

Cite this Article as: Kristian P Thaller (2015), "Agile in the management of scientific research collaborations", Journal of Administrative Sciences and Technology, Vol. 2015 (2015), Article ID 643806, DOI: $10.5171 / 2015.643806$ 
challenges and proposes a generalized strategy for managing Multidisciplinary Scientific Research Collaborations (MSRCs).The parallels between this management strategy and established Agile strategies are then drawn and discussed. The value to appreciating this link is considered in terms of defining a generalized approach to developing management strategies for MSRCs.

\section{Projects or programs?}

MSRCs combine the research work of distinct teams to achieve a common goal. As knowledge generated in any one 'subteam'is integral to the outputs of each of the others, close integration of the subteams is required both in terms of research activities and management. In most cases the common goal will have an associated budget meaning that there is a highly centralized financial and related management structure to the collaboration.This close integration is a feature of a project. In general the subteams involved in the collaboration will be drawn from established research groups. Though close cooperation between the sub-teams should be developed, their established nature tends towards a collaborative structure of parallel, interconnected sub-projects that are simultaneously driven by independent hierarchies and research interests with contribution to the shared objective being a device for securing funding. This structure is characteristic of a program. From this analysis it can be seen that MSRCs have some of the features of a project and some of a program. This highlights the fact that neither a standard project nor program management strategy can be applied to the collaboration. A successful management strategy must instead strike a balance between the methodologies in a single, fully integrated structure.

\section{Effect of knowledge generation}

In contrast to most programs, the targets in MSRCs are broadly based on scientific theory and reasoned assumptions. Even for programs that have clear target deliverables such as novel devices, there is always a coupled necessity for the generation of new knowledge. The scope of the MSRC may therefore be expressed in terms of the solutions to an evolving set of scientific questions that are focusing on delivering the level of knowledge required for a target solution. In effect the deliverable is the outcome of some combination of 'known unknowns' meaning that at any point in the project lifecycle the research scope is a 'well defined uncertainty'. The continual process of knowledge generation in a MSRC leads to an evolution in the understanding which was used to determine the research scope at the concept phase. Thus with each 'block' of generated knowledge the scientific questions that are the research scope need to be adapted to accommodate this new understanding. A successful management strategy has to be responsive to this generation of knowledge by employing an iterative approach to solving the questions that will deliver the program's target solution.

\section{Managing knowledge}

As discussed, the objective of a MSRC is the generation of new knowledge. A knowledge management strategy that effectively consolidates and shares this generated knowledge is an essential element for delivering synergy, research progress and effective decision making processes. In addition to the 'explicit' knowledge generated by the research outcomes, there is also a large body of 'tacit' knowledge to manage. Michael Polyani's statement "we know more than we can tell" (1966) is the widely recognized definition of tacit knowledge and how it can be distinguished from explicit knowledge. A substantial body of literature has since developed on the role that tacit knowledge plays in many fields and an insightful review of how tacit knowledge maps onto innovation management can be found in the conference paper by Seidler-de Alwis et al.,(2004). Of particular interest to MSRCs is their conclusion "that tacit knowledge management needs to be adapted quite closely to the innovation management of a firm for being able to achieve maximum innovation success". Although Seidler-de 
Alwis et al., analyzed innovation management from a commercial perspective, the same principles can be directly translated to MSRCs which have many of the features of consortia. In MRSCs team members are required to have highly specialized scientific expertise. The resultant diversity of the expertise poses a particular challenge to identifying the body of tacit knowledge, the first step toward developing a tacit knowledge management strategy. A successful management strategy has to consolidate both generated explicit knowledge and tacit specialist scientific knowledge as belonging to the collaboration rather than its default state of personal ownership.

\section{other challenges}

In addition to the specific challenges of MSRCs identified in the previous subsections, the management strategy must address all the standard (critical) success factors for a project or program (APM, 2012). The advanced, iterative nature of scientific research places particular emphasis on having a competent, committed team and maintaining motivation among the team members. The broad and multidisciplinary nature of the collaboration requires particularly strong internal communications processes. By addressing all of these challenges it is possible to develop a generalized strategy that can be used as the basis for managing large MSRCs.

\section{Leadership And Management}

Gibb (1954) first discussed the concept of two forms of team leadership: focused (i.e. a single leader) and distributed (i.e. shared leadership). At its most fundamental level the management structure of a MSRC must adhere to its collaborative nature. This means that it must respect the established hierarchies of the sub-teams as part of a mutual cooperation of (nominally) equal partners working together to deliver a shared goal.The multidisciplinary nature of the MSRC requires the expertise of all the research leaders for defining strategy. Their participation at the top level of management is also necessary for maintaining the positive engagement of sub-team leaders whose standing is already established outside the MSRC. Additionally there is also a growing body of evidence that distributed leadership has a positive effect on delivering a project's outputs (e.g. Carson et al., 2007 andD'Innocenzo et al., 2014). Extending substantial autonomy to the sub-teams while reaching strategic decisions collectively,i.e. adopting a program-like management strategy, offers the optimum distribution of responsibilities for the management of a large MSRC. A harmoniously functioning MSRC thus has a distributed management structure with a collective decision making and steering body as its top level of management. There are, however, significant risks carried when adopting such a distributed management structure and successful implementation of the management strategy requires these to be addressed.

\section{Achieving integration}

The first risk is that distributed management may result in poor integration of the sub-teams and a tendency towards dissociation and divergence of priorities. The established hierarchy of the sub-teams with its different levels of staff experience (i.e. professorial, postdoctoral or doctoral students) means that junior team members naturally look to their sub-team leader for direction. As this sub-team leader only represents one part of the MSRC, the importance of contributing to the shared goal and supporting the other sub-teams may not be appreciated by team members. This effect is exacerbated in the case of doctoral students by the sub-team leader's responsibility to enable them to complete a self-contained academic thesis. An individual who can provide visible leadership for the MSRC, separate from the distribution of management responsibilities, is thus important for building a well-integrated team working towards a clearly shared goal.

The need for visible leadership is directly linked to the benefits of developing an 'entity' that transcends the team members 
existing organizational loyalties. A shared identity that all team members can feel an equal part of serves to build a spirit of cooperation, enhances professional engagement across the team and maintains a continual awareness of the shared goals. A common identity can also act as a positive motivator by retaining a sense of ownership for collaborative achievements by those team members not directly involved in the completion step.

\section{Conflict resolution}

The second risk to distributed leadership is that there is an increased probability of and decreased ability to resolve management and strategy disagreements between the sub-team leaders. Although in a different context, the study by Boone and Hendriks (2009) reflects how integrating the work of team leaders with diverse interests and priorities may lead to a detrimental impact on the collaborative outcomes. Without executive authority residing in one individual, one of the imperatives on the team leaders to reach a decision of compromise is removed. Thus in the event of conflict the top level of management can become dysfunctional. Providing that the leader acts in the spirit of collaboration, appointing one sub-team leader to a position of executive authority can encourage the harmonious functioning of the collective decision making body and efficiently resolve issues.

\section{Independent management component}

As developed in the previous two sections, the collaborative senior management structure consists of three tiers. At the lowest level there are quasi-independent sub-projects led by established sub-team leaders. At the collaborative, program-like level the same sub-team leaders act as a collective decision making and steering body. Finally one of the sub-team leaders is granted executive authority to encourage the harmonious functioning of the program and to provide visible leadership for the team members. That these positions are all held by the research leaders is essential due to the specialist expertise that is required at all levels of decision making.

The risks of this distributed management structure are that: (i) any major collaborative program must implement a substantial body of program-level management that is not related to research expertise, and (ii) the same individuals are exercising management responsibilities at both sub-project and program levels leading to a potential conflict of interests. The supplementary management activities include: (i) developing and implementing the management strategy throughout the MSRC lifecycle, (ii) developing and implementing knowledge management and communication strategies, (iii) facilitating integration of the team, (iv) coordinating and scheduling the decision making processes, (v) resource monitoring and financial administration, and (vi) formal reporting and record keeping. To adequately address the scope of these activities requires a trained program manager who, by being independent of any of the sub-teams, can identify and help to mitigate any conflicts of interest.

In contrast to a normal project or program, in this analysis of the optimal management structure for a MSRC the decision making aspect has been separated from the other management responsibilities. Failure to synchronously exercise these two aspects of management will lead to a disjointed, ineffective management structure. Thus the program manager must be fully integrated to the collective decision making and steering body (Figure 1) and, as the program manager has no direct authority, the research leaders must be prepared to cooperate with the various management strategies and processes that need to be implemented. 


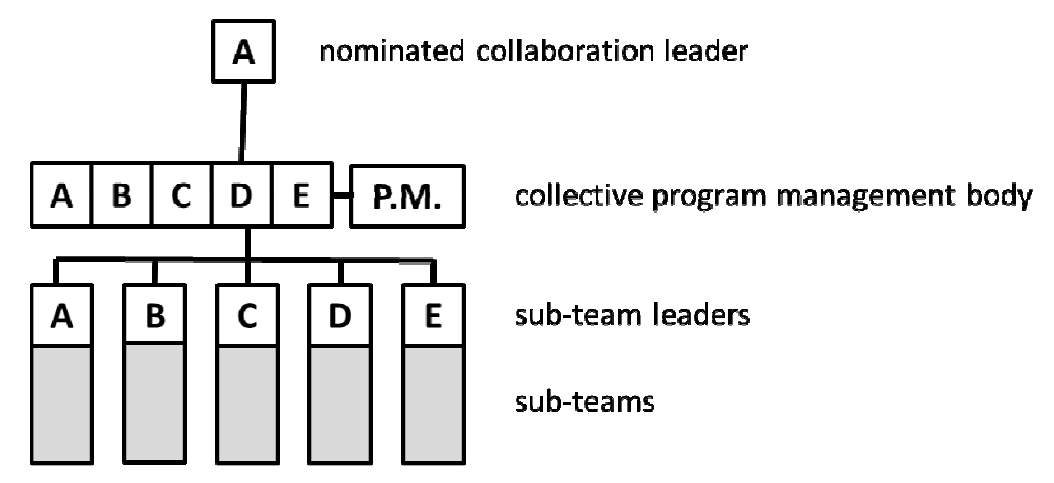

Figure1: The leadership and management structure for a successful MSRC that combines the work of five distinct research sub-teams. The sub-team leaders head the sub-projects and act as a collective decision making and steering body for the MSRC. Acting in the spirit of collective leadership, sub-team leader ' $A$ ' holds executive authority and is an identifiable leader for the common MSRC entity. At program level the decision makers (sub-team leaders) cooperate with an independent program manager for the exercise of the full spectrum of management responsibilities.

\section{The Agile Component}

Agile project management is a less hierarchical, iterative management technique that has been steadily growing in prominence, particularly in the field of software development (e.g. Fernandez \& Fernandez, 2009). An Agile management strategy will see responsibility delegated to appropriate members within an organization thereby creating a more responsive, collaborative management structure. As described by Wysocki (2006), there are three distinct yet similar Agile management strategies: Iterative, Adaptive and Extreme. This compares with the two traditional management strategies: Linear and Incremental. The three Agile management strategies are differentiated by how clear the solution is at the outset of the project. For an Iterative strategy a feedback loop is used to address issues on the path to a clear solution. For an Adaptive strategy the feedback loop is used to adjust the objectives of the next iteration, thus gradually converging on a solution. For an Extreme strategy the solution is identified during the course of the various iterations, sometimes referred to as 'goal searching'. Similarities and differences can be found when comparing the optimal management strategy for a MSRC to Agile management structures and methodologies.

\section{Management comparison}

The management and leadership structure described in section two in many ways mirrors the distribution of responsibilities under an Agile management strategy. In a fully Agile MSRC the responsibility for delivering different aspects of the shared objective would be fully delegated to the sub-teams and the role of the top-level decision making body would be limited. The level of management responsibility that is held by the single, albeit collective, decision making body in the developed MSRC strategy differentiates this approach from a fully Agile strategy. Rather it is a form of hybrid strategy, the advantages of which are well discussed in literature (e.g. Cunha \& Gomes, 2003 and Akgün et al., 2014). Effective delivery of short-term research goals requires decision making and information exchange at the level of the sub-teams, i.e. an Agile approach. Delivery of the shared, strategic goals requires the experience and direction of the senior management team, i.e. a traditional approach. This strategy returns to the program-like nature of the research collaboration with Agile management strategies being applied at sub-project level and traditional management strategies at program level. 


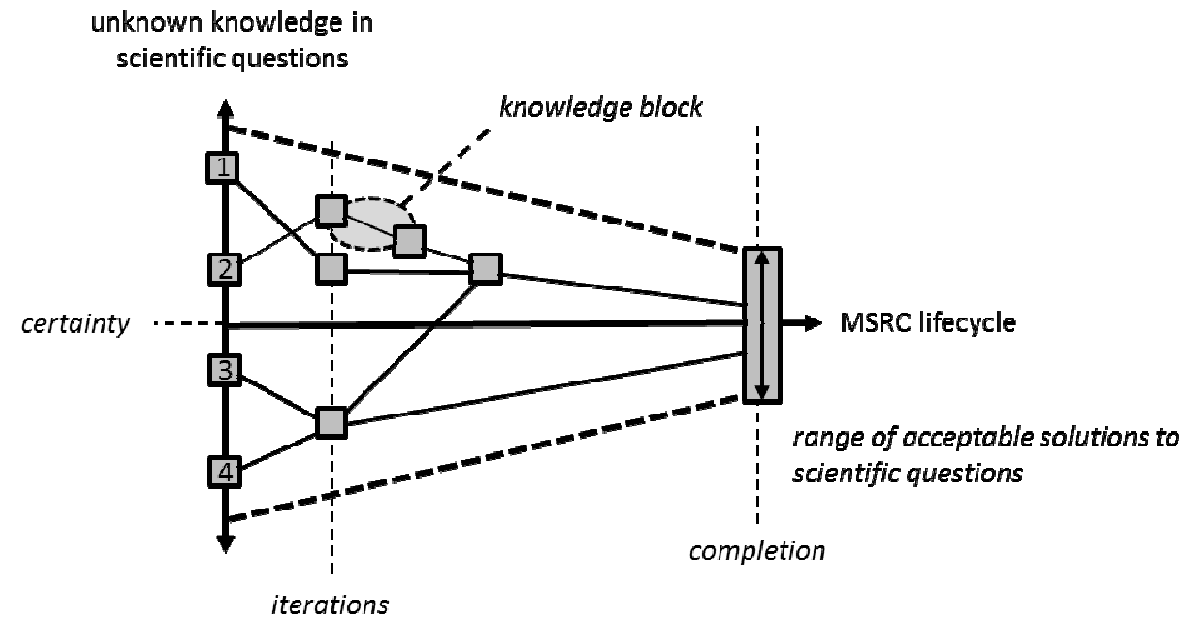

distinct scientific questions

Figure 2: A simplified plot of how the unknown knowledge in a MSRC reduces over the lifecycle to converge on a range of acceptable solutions to the scientific questions identified at the concept phase, i.e. Adaptive strategy. The figure shows the iterative nature of the research as each knowledge block is generated. The Extreme strategy employed for solving scientific questions two and three is shown by the increase in unknown knowledge over the first iteration. The essential sharing of knowledge across the entire collaboration is shown by the links between the scientific questions.

\section{Methodology comparison}

The requirement for new knowledge in a MSRC means that the target solutions to the scientific questions specified at the concept phase are goals rather than objectives. In other words even clear target deliverables are to a significant degree aspirational. Thus, the final solution will lie in some window for which the remaining unknowns have been reduced to a useful level, the exact details of the outcome depending on the solutions to the evolving set of fundamental scientific questions (Figure 2). Each block of generated knowledge redefines the scope of the scientific questions (i.e. the remaining unknown knowledge), a scope that should reduce as the research progresses and a final solution is converged upon. This feedback loop tending towards a final

target solution is a recognized feature of Agile methodologies.
The revision of the scientific questions with each knowledge block is managed in order to keep the program targeting its shared research goals. This is a feature common to the Adaptive method. However, although the collaborative strategy is revised to target the shared research objective, for individual scientific questions there may not be sufficient knowledge to predict within a reasonable degree of accuracy the solutions at the start of each iteration. Thus at the level of the individual knowledge blocks the research may in some cases be compared to the Extreme method. Therefore a MSRC is an integration of both the Extreme and Adaptive Agile methodologies. As with the management comparison this returns to the programlike nature of the research collaboration, Extreme strategies being applied at project level and Adaptive strategies at program level. 


\section{Value}

Recognizing the extent to which the described MSRC management strategy can be linked to existing Agile strategies is valuable because it relates the developed approach to well-established frameworks. A generalized approach to developing management strategies for MSRCs is thus derived. This is based on approaching the MSRC from the perspective of the described generalized management strategy. From this the Extreme, Adaptive and traditional components of the strategy can be identified. A suitable combination of well-tested strategies with readily available resources can then be applied to develop an integrated management structure that is responsive to the unique needs of a MSRC in an academic institution.

\section{Knowledge Management}

Knowledge management is recognized as a distinct, important field and there is a large body of supporting literature (e.g. Dalkir, 2005). Developing an effective knowledge management strategy is a critical success factor for implementing the broader management strategy described in sections two and three. In a MSRC there are three types of knowledge that need to be managed: experimental data, research outcomes (i.e. conclusions drawn from data) and tacit knowledge such as the intricacies of experimental procedure and underpinning scientific understanding. The discussions in sections two and three highlight the program-like nature of a MSRC. The degree of integration required for a program that is driven by the need for new scientific understanding results, however, in a project-like aspect to the management strategy.

\section{Collaborative knowledge}

The different degrees of detail in the knowledge required to facilitate various program activities creates a link between knowledge sharing and the various levels of the team structure. The sharing of knowledge can thus be broadly broken down into three levels: (i) researcher (/grassroots) level facilitating normal research activities, (ii) sub-team level to enable sub-team leader input (accountable manager), and (iii) program level to achieve synergy, form strategy and preserve knowledge. Implement a clear hierarchy for knowledge sharing can, however, lead to the loss of knowledge as it is condensed into a suitably transferrable form and any misunderstanding is perpetuated with each handover. There is therefore a requirement for all team members to actively engage in the sharing of knowledge at all levels. The reasons that each of the different types of knowledge in the MSRC must be shared are:

i) The relations between the data generated in one area of the collaboration and research in the others will not always be clear to the individual who has generated the data nor will the relations necessarily exist during the iteration in which the data is generated. Thus data needs to be pooled as a readily accessible resource for all team members at any point in the MSRC lifecycle.

ii) Research outcomes, with supporting data, need to be conveyed to the accountable sub-team leader who is coordinating the activities for solving a given scientific question. As they may influence other concurrent research activities as well as future iterations of the scientific questions, this requires them to also be conveyed to the collective team leadership and recorded for future reference.

iii) To support the multidisciplinary aspect of the MSRC, the underpinning scientific knowledge has to be transferred in a digestible format between team members (particularly leaders) who represent different specialisms.

iv) Tacit knowledge of experimental procedure has to be shared with any individuals performing similar tasks in order to standardize experimental procedures. This tacit knowledge also has to be identified and preserved somewhere within the project in the event that an individual leaves the collaboration. 
From this analysis it can be seen that a program driven by the need for new scientific understanding operates as a continually expanding 'knowledge pool' that all team members have to be able to access for the MSRC to be successful. The ability to readily identify required knowledge from this pool necessitates an effective knowledge management strategy. The integrated nature of this knowledge and interdependency that it creates between the sub-projects is the project-like feature of managing the research collaboration. The complexities of delivering a strategy that can achieve the knowledge sharing objectives highlights the value of an independent program manager in supporting the project.

\section{Communication driven structure}

An effective management structure that integrates the knowledge sharing considerations with the other aspects discussed in this article is to use a hierarchy of regular meetings (Figure 3) with supporting infrastructure such as: formalized reporting procedures, file sharing platforms and remote conferencing facilities. The structure described below has been successfully demonstrated in the context of the author's current program, a MSRC that unites thirty team members from six sub-teams across three sites in the United Kingdom.

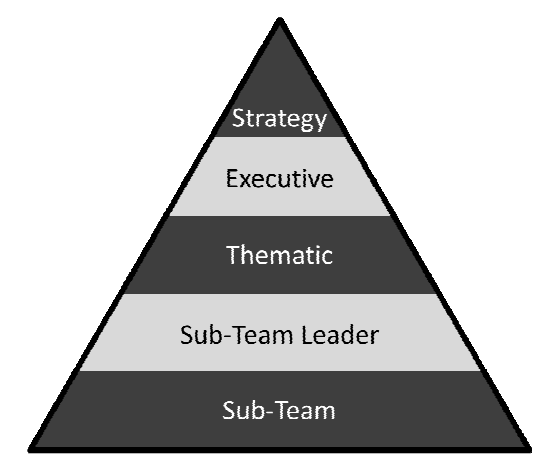

Figure.3: Meeting structure recommended for an effective MSRC knowledge management strategy. Sub-team meetings consolidate progress within the sub-projects. Sub-team leader meetings drive progress across the collaboration. Thematic meetings bring together all stakeholders in a research theme, thereby influencing the research direction and informing management decisions taken at Executive meetings. Strategy meetings are not part of the administrative framework and serve to enhance collaboration's synergy.

- Sub-team meetings: The lowest level of meetings should be held within each sub-team on a frequent (e.g. weekly) basis. They serve to consolidate progress and to maintain focus on the specific and shared goals. These are the only meetings not held at program level. Key stakeholders from other subteams may be included to add value when addressing points that have particularly strong program interdependencies.

- Sub-team leader meetings: The second level of meetings should be held with similar frequency to the sub-team meetings and are for the sub-team leaders to share progress reports separately to the formal decision making processes. The advantage of these meetings is that issues can be promptly identified and collaborative resources allocated to resolve these.

- Thematic meetings: The next level of meetings focuses on specific research themes (/scientific questions) that can be identified. These will evolve as the research scope reduces. The meetings should bring together all stakeholders in the theme, i.e. the team leaders and 
researchers both directly and indirectly impacted by the current state of the research. This means that individuals throughout the research pipeline can be involved in defining the research strategy for the theme, important for pre-empting issues. Those involved in delivering the theme's objectives can better appreciate the challenges faced by other researchers, reducing the risk of conflict situations. An awareness of the theme's state of progress helps stakeholders to efficiently schedule their other activities. For the author's program a monthly cycle of seven thematic meetings has been identified as effective for maintaining a responsive management strategy without placing an excessive management burden on the researchers. All team members should be contributing in some way to at least one of the themes. Thus the thematic meetings also serve to identify any researchers who are not being effectively used to contribute to the shared goals.

- Executive meetings: The top levels of administrative meetings are those of the collective leadership. These meetings are used to ratify the administrative actions of the program manager and, informed by the previous cycle of thematic meetings, to reassess the current iteration of scientific questions and maintain the program's strategic direction.

- Strategy meetings: The final set of meetings are for providing quarterly situation reports for all team members on all aspects of the MSRC, including those in which they are not stakeholders. This is essential to developing an understanding of the value of all members' contributions, nurturing a sense of shared ownership in the common goals, and building personal interactions between team members that strengthen informal channels of communication.
This structure for reporting and discussing research progress and outcomes means that all data and analyses are formally recorded and archived. The inclusion of input from all stakeholders, whether direct or indirectly via a lower-level meeting, at all levels of management minimizes the risk of losing tacit knowledge when reaching management decisions. This communication-centric structure thus addresses most of the knowledge sharing needs described in the previous subsection by supplementing the management strategies described in sections two and three.

Additionally this formal meeting and communication structure can be augmented by a multitude of accepted team development techniques. of particular importance to a research collaboration is 'shadowing', where one researcher directly observes the full scope of the work of another for a defined time period. This can be used to develop understanding between team members who have different specialisms. It can also be used to encourage standardization of experimental practices among individuals with the same specialism. Viewed more broadly, shadowing plays an important role in consolidating tacit knowledge as belonging to the team rather than being purely personal knowledge.

\section{Conclusion}

This article has identified the challenges specific to managing a large multidisciplinary scientific research collaboration in an academic institution. These can be summarized as being: (i) the hybrid project-program nature of collaborative research, (ii) the evolving understanding intrinsic to a project that is advancing the limits of scientific knowledge, and (iii) the challenges of managing the large body of generated explicit knowledge and significant tacit knowledge. The importance of having a management strategy that is responsive to these specific challenges has been highlighted and an effective management strategy that addresses these challenges described. The main features of this 
strategy are: (i) a program-level collective decision making and steering body composed of the sub-team leaders in full cooperation with an independent program manager, (ii) one sub-team leader nominated to have executive authority and provide visible leadership for a common, collaborative identity, and (iii) a formal communications structure inclusive of all stakeholders that is the basis of all decision making processes. This proposed strategy effectively integrates project/program management with knowledge management in a framework that can be translated to any MSRC. By balancing distributed and single leadership it consolidates the body of tacit knowledge in the MSRC and reduces the risk of dissociation and divergence of priorities between the established teams that constitute the MSRC. It drives progress by reducing administrative overheads while maintaining a high level of awareness of collective progress across the team hierarchy. This is the general contextual framework that can be used to develop a management strategy for any MSRC.

The parallels between the above management strategy and established Agile management strategies have been drawn and discussed. The proposed MSRC management strategy is identified as combining Adaptive and Extreme Agile strategies for the management of research, while retaining a traditional strategy for managing the strategic objectives. By identifying this hybrid structure formed of established project management strategies a generalized approach to developing effective management strategies for MSRCs in academic institutions has been presented. Approaching the MSRC from this perspective a project management professional can effectively integrate proven Agile and traditional techniques in accordance with best practice for the development of a tailored management strategy that is responsive to the central objective of scientific research, namely the generation of new knowledge.

\section{Acknowledgements}

The author is the Programme Manager for the SCFED Project (www.scfed.net), a multidisciplinary collaboration of British universities investigating the fundamental and applied aspects of supercritical fluids. The author gratefully acknowledges the EPSRC (United Kingdom) for funding this work.

\section{References}

1. Akgün, A. E., Keskin, H., Byrne, J. C. and Ilhan, Ö. Ö. (2014). Complex adaptive system mechanisms, adaptive management practices and firm product innovativeness. R\&D Management, 44: 18-41

2. Association for Project Management, (2012). APM Body of knowledge, $6^{\text {th }}$ edition. High Wycombe: Association for Project Management

3. Beaver, D. and Rosen, R. (1978). Studies in scientific collaboration. Part I. Scientometrics, 1: 65-84.

4. Beaver, D. and Rosen, R. (1979). Studies in scientific collaboration Part II. Scientometrics, 1: 231-245.

5. Boone, C. and Hendriks, W. (2009). Top management team diversity and firm performance: moderators of functionalbackground and locus-of-control diversity. Management Sci., 55: 165-180

6. Carson, J. B., Tesluk, P. E. and Marrone, J. A. (2007). Shared leadership in teams: an investigation of antecedent conditions and performance. Academy of Management J., 50: $1217-1234$

7. Cunha, M. and Gomes, J. (2003). Order and disorder in product innovation models. Creativity and Innovation Management,12: 174-187

8. Dalkir, K. (2005). Knowledge management in theory and practice. Oxford: Elsevier

9. Etzkowitz, H. and Leydesdorff, L. (1996). Emergence of a triple-helix of university-industry-government relations. Science and Public Policy, 23: 279-286

10. Fernandez, D. J. and Fernandez, J. D. (2009). Agile project management - 
agilism versus traditional approaches. $J$. Comp. Info. Systems, 49: 10-17

11. Gibb, C. A. (1954). Leadership. Handbook of social psychology, Reading MA.: Addison-Wesley,2: 877-917

12. Gibbons, M., Limoges, C., Nowotny, H., Schwartzman, S., Scott, P. and Trow, M. (1994). The new production of knowledge: the dynamics of science and research in contemporary societies. London: Sage

13. Godin, B. and Gingras, Y. (2000). The place of universities in the system of knowledge production. Research Policy, 29: 273-278
14. D'Innocenzo, L., Mathieu, J. E. and Kukenberger, M. R. (2014). A meta-analysis of different forms of shared leadership team performance relations. $J$. Management, Online Publication: DOI: 10.1177/0149206314525205

15. Polyani, M. (1966). The tacit dimension. Garden City N.Y.: Anchor Books

16. Seidler-de Alwis, R., Hartmann, E. and Gemünden, H. G. (2004). The role of tacit knowledge in innovation management. $19^{\text {th }}$ Annual IMP Conference, University of Lugano.

17. Wysocki, R. K. (2006). Effective software project management. Hoboken N.J.:Wiley Publishing Inc. 